\title{
COMPONENTES DEL INDICADOR DE DESARROLLO HUMANO (IDH): LA SITUACIÓN DE LAS PROVINCIAS DEL NORDESTE ARGENTINO EN LA DÉCADA DEL ’90.
}

\author{
PROF. VILMA LILIÁN FALCÓN (*) \\ Departamento de Geografía \\ Facultad de Humanidades. \\ Universidad Nacional del Nordeste (Argentina) \\ Avenida Las Heras $\mathrm{N}^{\circ} 727-$ \\ 3500 - Resistencia - Chaco - Argentina \\ Teléfono/FAX: $0054-3722-446958$
}

\section{RESUMEN:}

El presente trabajo fue realizado con el objetivo de lograr un diagnóstico de las provincias del Nordeste Argentino a partir del análisis de algunas variables relacionadas con los tres componentes principales del Indicador de Desarrollo Humano (IDH): educación, salud y trabajo.

Dado que nuestro propósito se centra en observar la situación de las cuatro provincias que conforman la región, el diagnóstico parte de considerar, en primer lugar, el contexto nacional para determinar la posición de estas provincias de acuerdo a cada variable analizada. En segundo lugar, el análisis se centra en el carácter intrarregional, es decir en el comportamiento espacial de algunas variables en el interior de cada provincia. En ambos casos nos valemos de la herramienta fundamental que brinda la representación gráfica y cartográfica.

La principal fuente de información fue el Censo Nacional de Población y Vivienda 1991 (INDEC) y las estadísticas provenientes de las Direcciones de Estadística y Censos provinciales que nos permitieron obtener datos más actualizados y en el caso específico del Indicador de Desarrollo Humano nos basamos en las estimaciones realizadas por el Programa Argentino para el Desarrollo Humano (PADH) en sus Informes 1995 y 1998.

\section{PALABRAS CLAVES:}

$<$ Desarrollo Humano $><$ Indicador de Desarrollo Humano $><$ Nordeste Argentino $><$ Situación Social $>$

\section{ABSTRACT: \\ COMPONENTS OF THE HUMAN DEVELOPMENT INDICATOR (HDI): THE STATE OF THE NORTH- EASTERN PROVINCIES IN ARGENTINA IN THE 1990'S}

This paper aims at sketching a diagnose of the north-eastern provinces in Argentina from the analysis of some variables related to the three main components of the Human Development Indicator (HDI): education, health and employment.

Since our purpose is to analyse the situation of the four that make up the region, the diagnose will consider first of all, the national context to determine the position of these four provinces as regards each variable. Secondly, the analysis is centred on interregional features of the region, that is to say the spatial behaviour of some variables in the interior of each province. In both cases we have used the invaluable information provided by graphic and cartographic tools.

The main source of information was the 1991 Population and Housing National Census (INDEC) and the statistics provided by the provincial Directions of Statistics and Census, that allowed us to use more up-dated data, and in the case of the Human Development Indicator, we have employed the estimates provided in the 1995 and 1998 reports by the Argentine Program for Human Development (PADH). 


\section{KEY WORDS:}

$<$ Human Development $><$ Human Development Indicator $><$ north-eastern $><$ social situation $>$

(*) E-mail: vfalcon@hum.unne.edu.ar

\section{COMPONENTES DEL INDICADOR DE DESARROLLO HUMANO (IDH): LA SITUACIÓN DE LAS PROVINCIAS DEL NORDESTE ARGENTINO EN LA DÉCADA DEL '90.}

El presente trabajo tiene como objetivo realizar una descripción del comportamiento que presentan los componentes principales del Indicador de Desarrollo Humano (IDH) en las provincias de Chaco, Corrientes, Formosa y Misiones (Nordeste Argentino).

Dado que nuestro propósito se centra en observar la situación de las cuatro provincias que conforman la región, se considera el contexto nacional para determinar la situación de estas provincias de acuerdo a cada variable analizada, valiéndonos de la herramienta fundamental que brinda la representación gráfica y cartográfica.

La principal fuente de información fue el Instituto Nacional de Estadística y Censos (INDEC) a través del Censo Nacional de Población y Vivienda 1991 y los Anuarios Estadísticos de la República Argentina (1991-1999) y, en el caso específico del Indicador de Desarrollo Humano, nos basamos en las estimaciones realizadas por el Programa Argentino para el Desarrollo Humano (PADH) en sus Informes 1995, 1996 y 1998.

\section{El concepto de Desarrollo Humano y su Indicador (IDH)}

EL Programa de las Naciones Unidas para el Desarrollo (PNUD) define al desarrollo humano como "el proceso de ampliar la gama de opciones de las personas, brindándoles mayores oportunidades de educación, atención médica, ingreso y empleo e incluso tiene que ver con el total de opciones humanas, desde un entorno físico en buenas condiciones hasta libertades políticas y económicas" (PNUD 1992:18). Sin lugar a dudas se trata de un concepto amplio e integral basado en la idea de bienestar de la población, que ayuda a distinguir entre dos aspectos del desarrollo humano: uno, es la formación de capacidades humanas como un mejor estado de salud o mayores conocimientos; el otro, es el grado en que los individuos emplean las capacidades adquiridas.

Esta concepción requiere que la medición del nivel de desarrollo humano de un determinado país, comunidad o grupo social, no se base solamente en componentes económicos que, aunque también son importantes considerar, constituyen una aproximación incompleta dado la complejidad del proceso señalado. Dentro del esquema propuesto por el PNUD se procura enfatizar en la gran divergencia existente entre niveles de riqueza material y de desarrollo humano. Por esta razón, el principal objetivo subyacente en la construcción del IDH es proporcionar referencias cuantitativas de las privaciones humanas y de las distancias existentes con respecto a metas posibles de alcanzar y monitorear la eficacia de las políticas en curso.

El IDH es un índice compuesto que toma en cuenta tres elementos básicos, dos de los cuales aluden a la formación de capacidades (longevidad y nivel de conocimientos) y un 
diferencial para algunas provincias del Norte cuyo índices las ubica en la categoría de

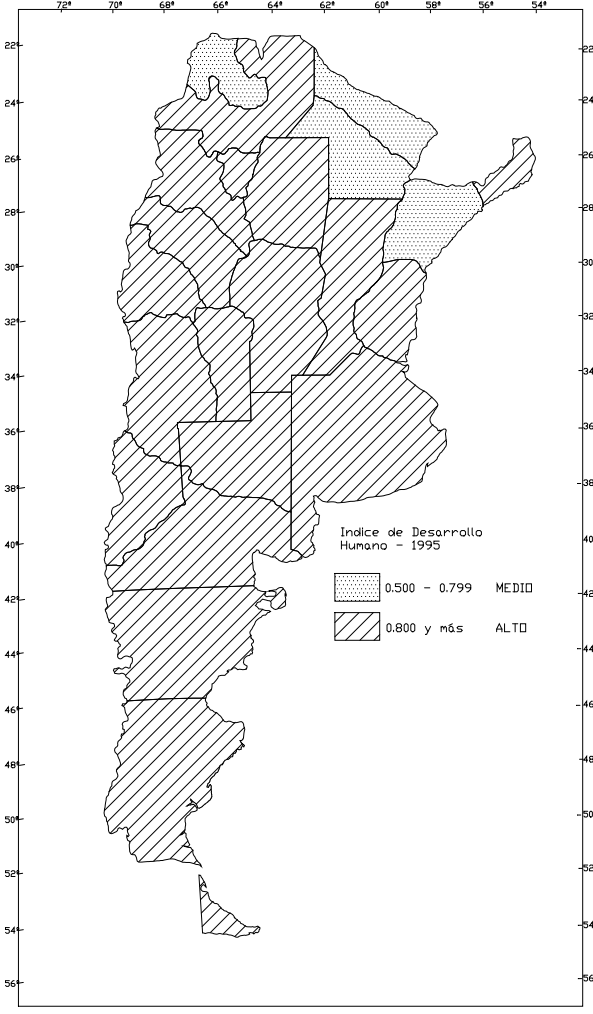

desarrollo humano medio.

En el cuadro $\mathrm{N}^{\circ} 1$ podemos observar el valor de IDH que le corresponde a cada provincia y, al mismo tiempo, la posición que ocupa cada una de ellas en el conjunto nacional; destacándose la ciudad de Buenos Aires (distrito Capital) seguida por Córdoba y Tierra del Fuego. En contraposición, ocupando los últimos lugares se encuentran las provincias del Norte, en las que se destacan por su marginalidad las provincias de Chaco, Formosa y Corrientes (NEA) y Jujuy (NOA).
Cuadro N 1: ARGENTINA, IDH estimado para1995.-

\begin{tabular}{|r|r|r|}
\hline $\begin{array}{r}\text { PROVINCIAS o } \\
\text { Distrito }\end{array}$ & $\begin{array}{r}\text { I.D.H. } \\
\text { estimado } \\
\text { para 1995 }\end{array}$ & Posición \\
\hline Ciudad de Bs. Aires & 0.923 & 1 \\
\hline Córdoba & 0.896 & 2 \\
\hline Tierra del Fuego & 0.895 & 3 \\
\hline Buenos Aires & 0.894 & 4 \\
\hline Santa Fe & 0.891 & 5 \\
\hline Santa Cruz & 0.888 & 6 \\
\hline Mendoza & 0.886 & 7 \\
\hline La Pampa & 0.883 & 8 \\
\hline La Rioja & 0.879 & 9 \\
\hline Neuquén & 0.878 & 10 \\
\hline Entre Ríos & 0.876 & 11 \\
\hline Chubut & 0.876 & 12 \\
\hline San Luis & 0.870 & 13 \\
\hline Río Negro & 0.873 & 14 \\
\hline San Juan & 0.873 & 15 \\
\hline Catamarca & 0.850 & 16 \\
\hline Misiones & 0.840 & 17 \\
\hline Tucumán & 0.828 & 18 \\
\hline Salta & 0.813 & 19 \\
\hline & &
\end{tabular}




\begin{tabular}{|r|r|r|}
\hline Corrientes & 0.791 & 20 \\
\hline Jujuy & 0.763 & 21 \\
\hline Chaco & 0.797 & 22 \\
\hline Santiago del Estero & 0.824 & 23 \\
\hline Formosa & 0.778 & 24 \\
\hline Total del país & $\mathbf{0 . 8 8 7}$ & \\
& & \\
\hline
\end{tabular}

Sin dudas lo que el IDH refleja para nuestro país, es producto de determinadas situaciones sociales, demográficas, económicas y culturales de cada provincia; es por ello que, a los efectos de mostrar algunos de estos aspectos, se representó una secuencia de mapas con los principales componentes del indicador. En esa serie de Mapas los indicadores referidos a la situación educativa, a la salud e Ingreso per cápita, permiten advertir las particularidades que se exponen a continuación.

\section{$>$ Respecto de la variable conocimiento:}

En general la situación de nuestro país en la década del '90, indica que el porcentaje de matriculación alcanza a por lo menos el $97 \%$ de los niños en edad escolar, porcentaje que se reduce al $80 \%$ si se considera la matriculación combinada (primaria, secundaria y terciaria).

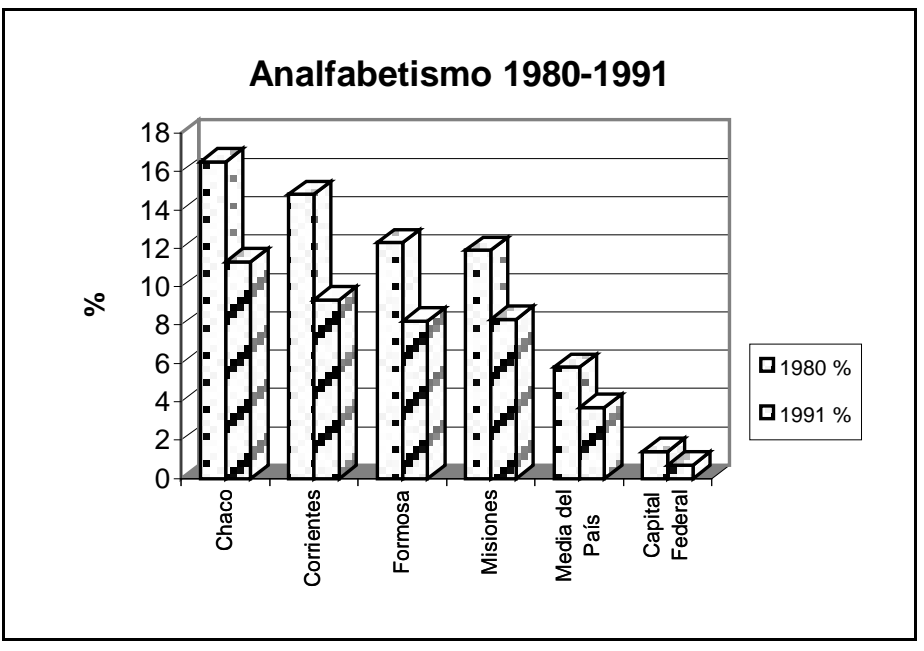

Estas cifras no hacen más que confirmar los logros que se dieron en nuestro país en lo referido a la educación en las últimas décadas.

El Gráfico $\mathrm{N}^{\mathrm{0}} 1$ permite advertir las condiciones de analfabetismo de la población de más de 10 años de las provincias del NEA. En el mismo se observa una tendencia positiva ya que los valores muestran una disminución desde 1980 a 1991, hecho que se corresponde con la situación a nivel nacional, también representada.

En efecto, los valores medios de analfabetismo del país indican que la proporción de población analfabeta ha disminuido gradualmente, ya que del 6\% observado en 1980 se pasa en 1991 a un $3,7 \%$.

No obstante ello, las diferencias regionales son bastante marcadas y ello se evidencia en los valores que le corresponden a las cuatro provincias en estudio que, en promedio, oscilan en alrededor de un $11 \%$ de población analfabeta para el año 1991, es decir que prácticamente triplican a la media nacional.

En la Serie $\mathbf{N}^{\mathbf{0}} \mathbf{1}$ del Anexo Cartográfico se puede ver que, efectivamente, dos de las provincias del NEA -Chaco y Corrientes- cuentan con las mayores tasas de analfabetismo. Las diferencias adquieren aún mayor relevancia si se comparan las cifras correspondientes a la Capital Federal, cuyo valor porcentual $(0,7 \%)$ es, por ejemplo, 18 veces menor al que presenta la provincia del Chaco (12.3 \%) y 14 veces más baja que la provincia de Corrientes $(10,3 \%)$.

Por otro lado, el promedio de años de escolaridad (1991) representa, para Chaco, Formosa y Misiones, los valores más bajos que se observan a nivel nacional, y están indicando que gran parte de la población de estas provincias no alcanza a recibir los 7 años de 
escolarización primaria que contempla el Sistema Educativo Formal. Por otro lado, el mapa que representa la proporción porcentual de alumnos matriculados en todos los niveles en el país, permite advertir que solo cuatro provincias superan el 4\%, destacándose Buenos Aires, seguida por Córdoba, Mendoza y Santa Fe.

Las condiciones más marginales de las provincias del Norte del país quedan expuestas en el mapa que representa la proporción porcentual de niños sin asistencia escolar y que además forman parte de hogares con Necesidades Básicas Insatisfechas (NBI), ${ }^{2}$ registrándose en la provincia del Chaco el porcentaje más alto.

Es interesante destacar estas diferencias, ya que dan cuenta de los desequilibrios regionales que se dan en nuestro país y, más aún cuando algunos indicadores relacionados con el nivel cultural y educacional colocan a la Argentina en una posición relativamente sobresaliente, no solo a nivel latinoamericano sino también mundial.

\section{$>$ Respecto de los indicadores del nivel de salud}

Cuando se intenta analizar las condiciones de salud de un grupo poblacional, frecuentemente se utilizan indicadores que intentan medir la ausencia o presencia de enfermedad, tal es el caso de la Esperanza de Vida y la Mortalidad (General, Infantil, Neonatal, etc.). En este caso, para observar la situación regional en el contexto nacional se analizan tales indicadores (Ver Serie $\mathbf{N}^{\mathbf{0}} \mathbf{2}$ del Anexo Cartográfico), pero además se consideran otros que guardan relación con la variable que se pretende describir.

\section{- La Mortalidad Infantil}

La Tasa de Mortalidad Infantil expresa el número de niños que mueren anualmente antes de cumplir el año; los valores se expresan cada mil nacidos vivos en el período considerado y constituye uno de los principales indicadores del nivel socioeconómico de un país o región. En el contexto latinoamericano, la Argentina ocupa una posición favorable como lo reflejan las estadísticas del Cuadro №3, aún así las tasas todavía son elevadas si se las compara con los logros alcanzados por otros países como por ejemplo Australia ( 8\%) o Nueva Zelanda (9\%o).

Cuadro $N^{\circ}$ 3: TMI en algunos países latinoamericanos

\begin{tabular}{|c|c|}
\hline País & TMI en \%o \\
\hline Argentina & 25 \\
\hline Bolivia & 83 \\
\hline Brasil & 58 \\
\hline Chile & 17 \\
\hline Paraguay & 35 \\
\hline Uruguay & 21 \\
\hline
\end{tabular}

\footnotetext{
${ }^{2}$ Según lo define el INDEC, se consideran Hogares con NBI aquellos en los cuales está presente al menos uno de los siguientes indicadores de privación:

Hogares que habitan viviendas con más de tres personas por cuarto (hacinamiento crítico)

Hogares que habitan una vivienda de tipo inconveniente (pieza de inquilinato, vivienda precaria u otro tipo)

Hogares que habitan en viviendas que no tienen retrete o tienen retretes sin descarga de agua

Hogares que tienen algún niño en edad escolar que no asiste a la escuela

Hogares que tienen 4 ó más personas por miembro ocupado y en los cuales el jefe tiene bajo nivel de educación (solo asistió dos años o menos al nivel primario)
} 
Fuente: Banco Mundial, Informe Sobre el Desarrollo Mundial.1993

En nuestro país, la T.M.I., al igual que la Mortalidad General, ha registrado un continuo descenso en las últimas décadas, como se puede observar en el gráfico siguiente.

\section{Gráfico $N^{\circ} 2$}

ARGENTINA. Tasa de Mortalidad Infantil.

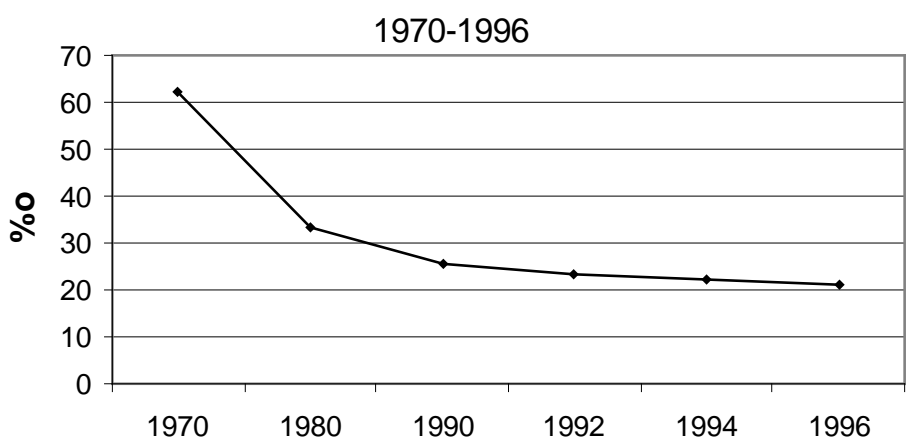

En general, estos logros suelen ser atribuidos a los adelantos científicos que permitieron la difusión masiva de antibióticos y vacunas, a lo que se suma la ampliación en la cobertura de los servicios sanitarios, tanto públicos como privados y a la extensión en la provisión de los servicios de agua potable.

Elaboración propia en base a:

INDEC, 1998. Anuario Estadístico de la República Argentina, Bs.As.

Precisamente, un elemento que juega un papel muy importante en el nivel sanitario de la población es la disponibilidad de agua potable, aspecto que se considera importante dada las características climáticas de las provincias en estudio, donde las altas temperaturas estivales provocan con frecuencia, elevados casos de deshidratación, hecho que se constituye en una de las principales causas de la mortalidad de la población, tanto en edades tempranas como en las muy avanzadas. Por otro lado, la calidad del agua que se les brinda a los niños es un factor de consideración ya que suele estar asociado -junto con una mala alimentación- a las “infecciones intestinales”, una de las principales causas de muerte infantiles. ${ }^{3}$

Es por ello que se representó en el Gráfico $\mathrm{N}^{\circ} 3$ el porcentaje de población cubierta con servicios de agua potable.

\section{Gráfico $N^{\circ} 3$}

Población cubierta con servicio de agua potable (en porcentaje) Año 1991

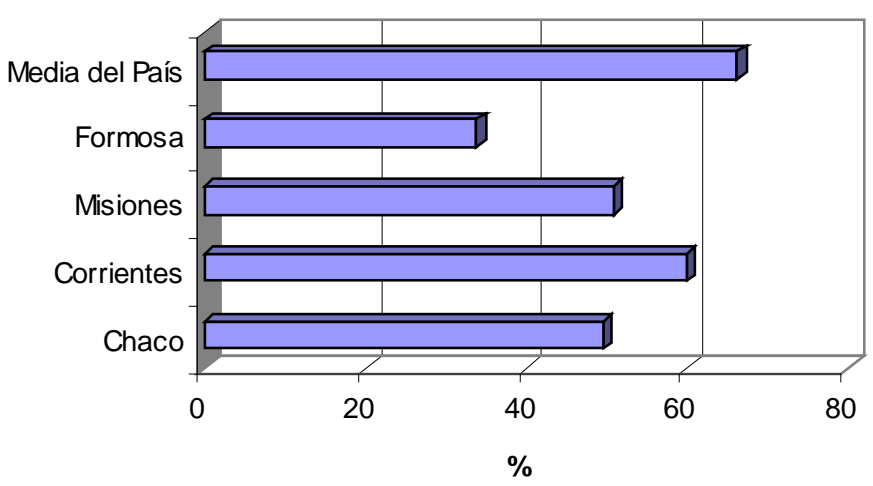

Elaboración propia en base a:
Si tenemos en cuenta los promedios provinciales, las cuatro provincias presentan valores inferiores a la media nacional (66,2\%), aunque la provincia de Corrientes se acerca bastante a ésta; en el otro extremo, la provincia de Formosa solo tiene, en promedio, alrededor del $20 \%$ de su población cubierta con este servicio.

\footnotetext{
${ }^{3}$ Ramírez, Mirta Liliana (1995:183)
} 
Si se analiza el mapa correspondiente a la Tasa de Mortalidad Infantil en la Argentina, año 1993, (Ver serie $\mathbf{N}^{\mathbf{2} 2}$ en Anexo Cartográfico) se observa que el Norte y Oeste del país son las regiones cuyo valores sobrepasan a la media nacional (22,9\%o), resultando también en este aspecto el área más desfavorecida del país. En este sentido, las provincias del Nordeste, especialmente Chaco y Formosa, presentan las condiciones más críticas (34,4 y 31,4 \%o respectivamente).

\section{- La Esperanza de Vida}

La Esperanza de Vida al nacer, representa el promedio de años que viviría una persona en un lugar determinado, o expresado de otro modo "el promedio de años que vivirá cada componente de una generación de recién nacidos que estuviera toda su vida expuesta al nivel de mortalidad representado por una tabla de vida" Somoza, (1971) ${ }^{4}$.

El Programa de las Naciones Unidas incluye a la esperanza de vida al nacer como uno de los componentes esenciales para determinar el Indice de Desarrollo Humano, sustentándola en la consideración de que éste puede darse en tanto las personas puedan lograr "vidas largas y saludables". PNUD, (1991)

En nuestro país, la expectativa de vida ha evolucionado en forma constante, pasando de 67,4 a inicios de la década del 70, a los 72,6 años según datos correspondientes a 1994. Esta evolución puede ser observada a través del siguiente gráfico en el que se incorporan además, las cifras proyectadas hasta el año 2000.

\section{Grafico $N^{\circ} 4$}

\section{Argentina. Esperanza de Vida al nacer} $1960-2000$

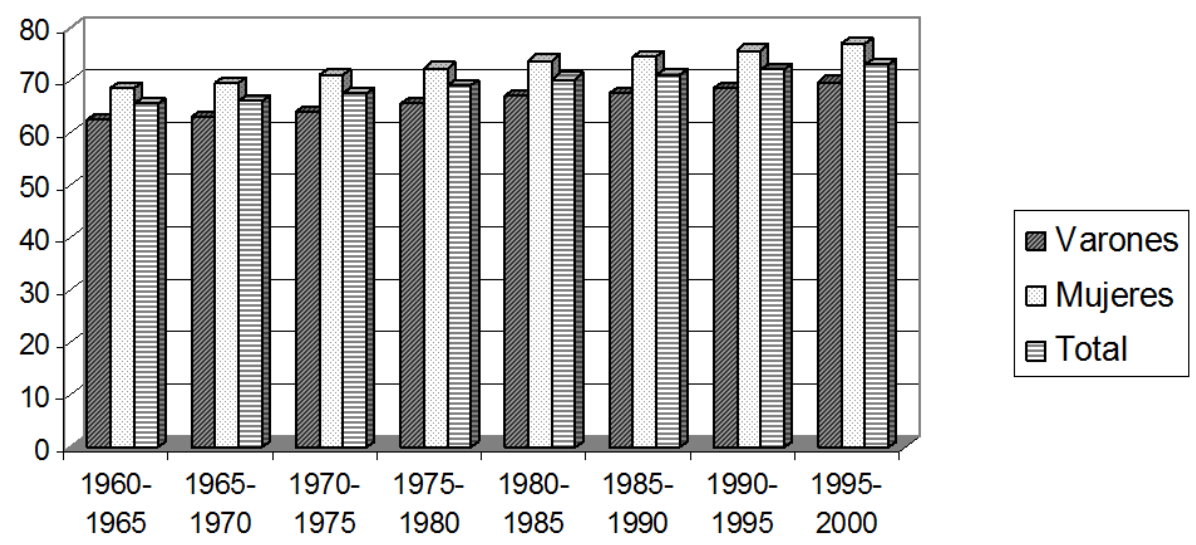

Elaboración propia en base a:

INDEC, 1998. Anuario Estadístico de la República Argentina, Bs.As.

Podemos advertir que el grupo femenino es quien cuenta con mayores expectativas de vida, alcanzando los 75,7 años en el quinquenio 1990-1995 por sobre los 68,6 que presenta el grupo de los varones. 
En general, la Esperanza de vida que tiene actualmente la Argentina no está muy alejada de los valores que ostentan los países más desarrollados como por ejemplo Japón, Estados Unidos o Suiza (79, 76, 78 años respectivamente).

Analizando la situación a nivel nacional, la representación permite advertir que las provincias del centro del país se destacan por presentar los mayores valores, en contraposición con la situación que reflejan las provincias del Norte, en las cuales Chaco y Formosa tienen las cifras más bajas de esperanza de vida - inferior a los 70 años- junto con las provincias de Salta y Jujuy, es decir, por debajo del promedio nacional que llega los 72, 6 años.

Si comparamos el comportamiento de esta variable en el grupo de las provincias del Nordeste, a través del gráfico $\mathrm{N}^{\circ} 4$ podemos observar que la población de Corrientes y Misiones tienen mayores expectativas de vida y se acercan más al valor medio del país. Si se discriminan los valores por Sexo, también aquí se sigue la tendencia general del país, que indica que las mujeres viven al menos 6 o 7 años más que los hombres.

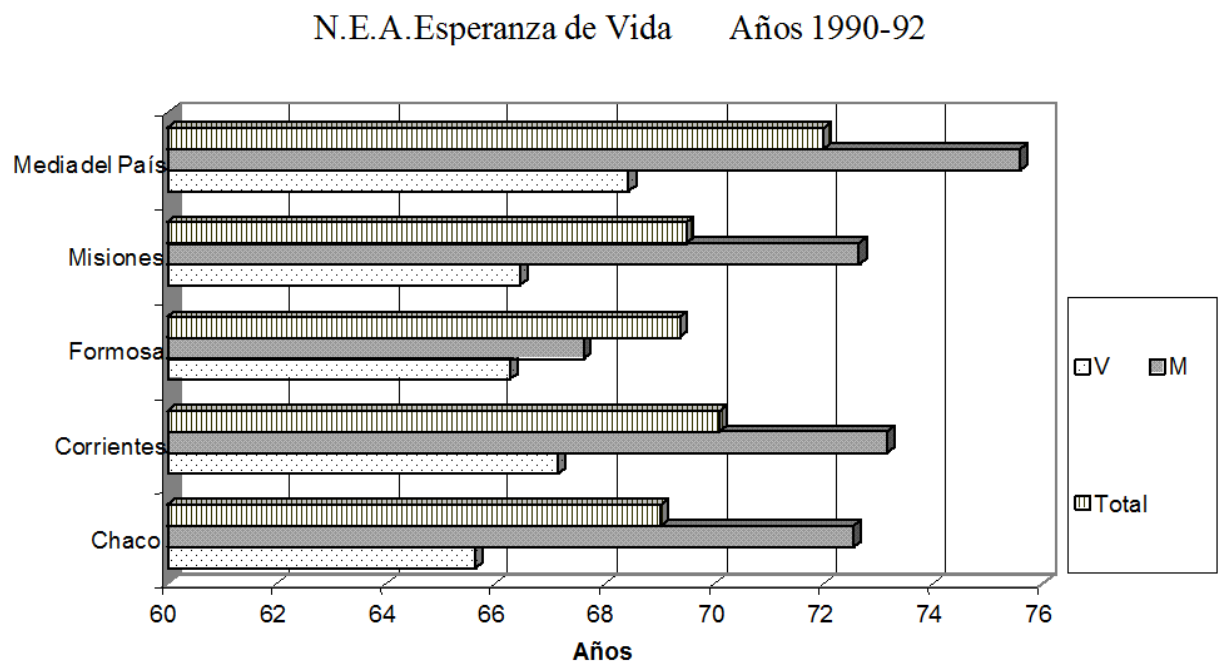

Elaboracion propia en base a:

INDEC-CELADE 1995. Serie Análisis Demográfico 4 y 5. Ministerio de Salud y Acción Social. Programa Argentino de Estadísticas.

Otro de los aspectos que interesa destacar es la posibilidad o restricción que tiene la población para acceder a los servicios de salud. El mapa que representa la proporción porcentual de población sin acceso a Obra Social ni Mutual muestra que para el año 1991, gran parte del país cuenta con valores elevados de población sin ese servicio; y nuevamente las provincias del Norte ostentan con las más elevadas proporciones, siendo las provincias de Chaco y Formosa las que presentan las peores condiciones (51,2\% y 56.4\% respectivamente).

A modo de síntesis, el mapa correspondiente al Indice de privación en salud resume claramente la situación sanitaria de la población de las provincias consideradas.

\section{- Respecto de las variables relacionadas con el ingreso}


La perspectiva del Desarrollo Humano señala a la economía como generadora de la oportunidad del trabajo, del ingreso y del acceso a los mercados. El trabajo, en tanto forma

ARGENTINA. Tasa de empleo y desocupación

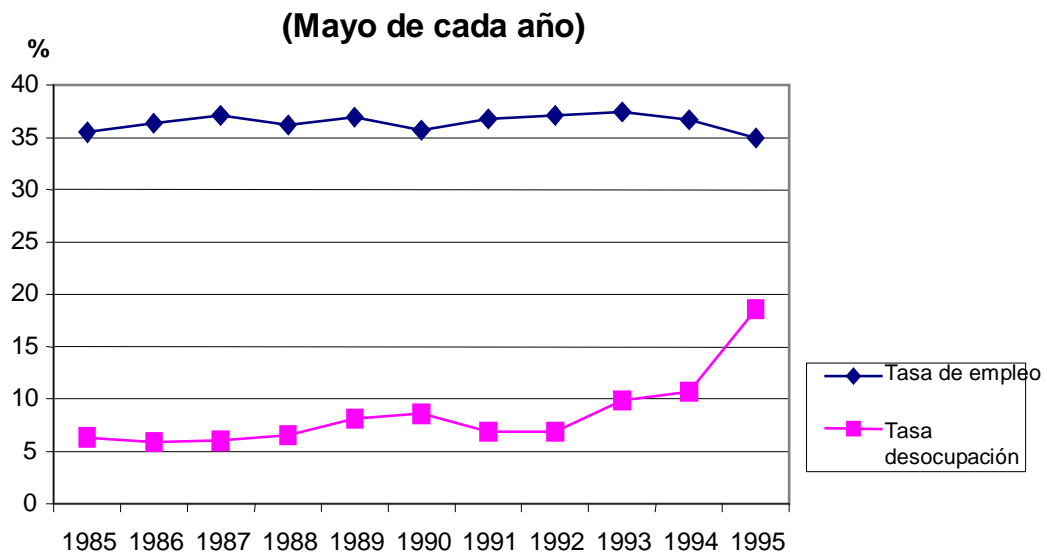

Elab. propia en base a INDEC- Anuario Estadístico de la Rep. Argentina

prioritaria de participación en la generación y apropiación del producto social y el modo de acceso a los mercados de bienes y servicios, son instrumentos fundamentales de la articulación del sistema productivo. La falta o carencia de trabajo desvanece las posibilidades del ingreso y acceso a los mercados. En ese sentido, es interesante destacar que la Tasa de desocupación en la Argentina ha variado en el período 1985-1995 en un 12,3\%, observándose la correspondencia inversa con la línea evolutiva referente a la tasa de empleo.

Si nos basamos en la representación cartográfica del ingreso por habitante para el año 1995 (Serie No 3 del Anexo cartográfico), observamos claramente las disparidades regionales ya que nuevamente las provincias del Norte se destacan por poseer los valores más bajos registrados a nivel nacional, por el contrario en las provincias del centro y sur del país se observan mejores condiciones de ingreso de sus habitantes. Por otro lado, el análisis del índice de privación del ingreso refleja claramente la situación marginal de las provincias del norte Argentino, más aún de las provincias de Chaco, Formosa y Corrientes.

Se consideró conveniente representar en esta serie los valores del Indice de Desarrollo Infantil (IDI) ya que se trata de un indicador que refleja la situación de la infancia y, en forma indirecta, las condiciones de oportunidades y potencialidades que tiene la Sociedad. En forma comparativa, un alto I.D.I. generalmente está asociado con un alto Desarrollo Humano, es decir que debe haber una correlación positiva entre ambos valores. En nuestro país esa correlación no se da, ya que como se puede observar en el mapa de la Serie, el valor del I.D.I. para el país es de 0,767 mientras que el I.D.H. es de 0,887. Esto estaría indicando, según las consideraciones del P.A.D.H., una regresión potencial que refleja el retraso de las políticas y estrategias hacia la infancia, o si se quiere, un atraso en las inversiones hacia el futuro. (cfr. P.A.D.H. 1996:233).

\section{A MODO DE CONCLUSION}

Hemos observado, a través del análisis de los tres componentes principales del Indicador de Desarrollo Humano, que las provincias del Nordeste Argentino presentan, en conjunto, características de marginalidad social, ya que los indicadores analizados se dan en 
valores a veces muy alejado de los promedios nacionales y en algunos casos de otras provincias o distritos; esto hace que junto con las vecinas provincias del Noroeste sean consideradas como el área del país que refleja las menores condiciones sociales para el desarrollo de su población.

De acuerdo a lo observado en lo referido a la situación educativa no podemos negar que contar con un promedio de alfabetismo del 97\%, ó un promedio de 8,13 años de escolaridad, es indicativo de los logros que se fueron dando en el Sistema educativo en nuestro país. Aún así es de esperar mayores mejoras si consideramos el proceso de transformación que comenzó a darse en la década del 90, especialmente en lo que hace al aumento de años de escolaridad básica obligatoria operado en el marco de la implementación de la Ley Federal de Educación $^{5}$. Si bien el análisis se basó en indicadores de principio de la década del 90, los datos del próximo Censo Nacional de Población darán cuentas de los avances y resultados concretos.

No obstante ello, tampoco podemos negar que algunas provincias de nuestro país lejos están de alcanzar los promedios mencionados y, como hemos observado al tratar este tema, las provincias del Norte son las que más se encuentran alejadas del referente nacional, a la vez que presentan altos índices de Privación en Educación.

Si tenemos en cuenta que el acceso a la educación general básica constituye un avance en la generación de igualdad de oportunidades, debemos concluir que un gran porcentaje de la población del Nordeste carece de esta oportunidad, ello también supone una mayor marginación de la población que habita estos espacios ya que las demandas de la sociedad actual, especialmente en el área laboral, exige cada vez más y mejores conocimientos.

Por otro lado, el análisis de las variables relacionadas con la situación sanitaria, guardan mucha similitud con lo mencionado para educación. Si bien la expectativa de vida de la población argentina es alta -72,4 años-, existen áreas, entre ellas las provincias de Chaco y Formosa, con una esperanza de vida que no llega a los 70 años.

En general, los datos que hemos manejado para el análisis de algunos aspectos relacionados con la situación sanitaria en las cuatro provincias, revelan la gravedad de la situación para la población de esta parte del país y más aún si a ello agregamos algunos indicadores de la situación laboral, ya que al crecer el ritmo de la desocupación y subocupación, se genera un deterioro de la capacidad de acceso a los servicios de las obras sociales o mutuales por parte de la población. Este hecho deriva a su vez en una gran demanda hacia el sector público de la salud que, en la mayoría de los casos, se encuentra con grandes restricciones de recursos y se ve superada por una demanda cada vez mayor. Por otro lado, se trata de una población con poca capacidad de recursos como para acceder a la medicina privada.

En síntesis, al tratarse de provincias empobrecidas con pocos recursos para enfrentar la crisis económica actual, se deriva necesariamente en una disminución de las condiciones sociales de la población. Aún cuando existe un compromiso a nivel gubernamental por mejorar los índices de Mortalidad Infantil y Esperanza de vida, la compleja trama de la realidad socioeconómica de estos espacios requieren de un refuerzo en la implementación de las políticas sociales, pero sus formas de aplicación no solo debe apuntar a cubrir las necesidades más básicas de la población, que de hecho deben ser prioridad, sino que además deberían intentarse cambios estructurales de los sistemas existentes, especialmente en los

\footnotetext{
${ }^{5}$ La nueva estructura aprobada a partir de la Ley Federal de Educación N N 24.195, crea una Educación General Básica de 9 años (desde los 6 a los 14) e incorpora el nivel inicial (5 años) como obligatorio para el cursado de la escuela primaria.
} 
referidos a la salud y a la educación, de manera que en conjunto se alejen de las características típicamente asistencialistas y pasen a ser políticas de promoción humana.

Un adecuado control del Estado -nacional, provincial, municipal- en lo referente a la calidad de las prestaciones que se brinda en ámbitos ya descentralizados, puede compensar las desigualdades regionales que produce precisamente ese tipo de política. Pero no es menos importante considerar la participación de la Sociedad Civil como verdaderos actores sociales, quienes desde las funciones específicas, realidades, residencias, estratos sociales, podamos diagnosticar, promover y proponer alternativas de desarrollo.

\section{BIBLIOGRAFIA}

- AGUILAR, Armando (1999). Desarrollo regional en la agenda de gobierno: Algunas implicaciones de políticas públicas. En: Indicadores Económicos. Departamento de Economía y Finanzas, Facultad de Ciencias Económicas. UNNE, Julio.

- ALFONSO, Elena (1999). Las regiones Argentinas y el comercio exterior. En: Indicadores Económicos. Departamento de Economía y Finanzas. Facultad de Ciencias Económicas. UNNE. Julio de 1999, $\mathrm{N}^{\circ} 38$.

- BESIL, Antonio (1995).-Chaco: Recursos, empleo y obras Públicas provinciales. En: Indicadores Económicos. Departamento de Economía y Finanzas, Facultad de Ciencias Económicas. UNNE. Año 4, No 22. Mayo.

- BESIL, Antonio (1995) Crisis financiera global de las provincias argentinas. En: Indicadores Económicos. Departamento de Economía y Finanzas, Facultad de Ciencias Económicas. UNNE. Año 4, Nº 22. Diciembre de 1995.-

- BLUMER-THOMAS, Victor (1998). La Historia Económica de América Latina desde la Independencia. Fondo de Cultura Económica, México., D.F.

- CARLEVARI, Isidro J.F. (1983) Compendio de Geografía Económica Mundial y Argentina. Editorial ERGON, Buenos Aires.

- COMISION ECONOMICA PARA AMERICA LATINA Y EL CARIBE (CEPAL). El Regionalismo abierto en América Latina y El Caribe al servicio de la transformación productiva con equidad. Naciones Unidas, Chile. 1994.

- CONESA, Eduardo R. (1996). Desempleo, Precios relativos y Crecimiento Económico. Ediciones Depalma, Buenos Aires.

- CUNILL GRAU, Nuria (1997). Repensando lo público a través de la Sociedad. Nuevas formas de gestión pública y representación social. Centro Latinoamericano de Administración para el Desarrollo (CLAD), Nueva Sociedad Editores, Caracas, Venezuela. 
- ISUANI, Aldo, FILMUS, Daniel (Compiladores). La Argentina que viene. Análisis y propuestas para una sociedad en transición. Grupo Editorial NORMA S.A. -UNICEFFLACSO. Buenos Aires, 1998.-

- MONTOYA, Silvia y OLIVERO, María P. (1998). Claves para reducir la pobreza agregada disminuyendo las disparidades regionales Argentina 1990-1997. En: Revista Estudios.IERAL- Fundación Mediterránea. Año XXI, No 85, Abril-Junio.

- POGRAMA ARGENTINO PARA EL DESARROLLO (PADH). Informe Argentino para el Desarrollo, 1995. Honorable Senado de la Nación. Comisión de Ecología y Medio Ambiente. Buenos Aires.

- PROGRAMA ARGENTINO PARA EL DESARROLlO (PADH). Informe Argentino para el Desarrollo, 1996. Honorable Senado de la Nación. Comisión de Ecología y Medio Ambiente. Buenos Aires, 1996

- PROGRAMA ARGENTINO PARA EL DESARROLLO (PADH). Informe Argentino para el Desarrollo, 1997. Tomo I y II .Honorable Senado de la Nación. Comisión de Ecología y Medio Ambiente. Buenos Aires, 1997

- PROGRAMA ARGENTINO PARA EL DESARROLLO (PADH). Informe Argentino para el Desarrollo, 1998. Tomo I y II. Honorable Senado de la Nación. Comisión de Ecología y Medio Ambiente. Buenos Aires, 1998

- PROGRAMA DE LAS NACIONES UNIDAS PARA EL DESARROLLO (PNUD). Desarrollo Humano. Informe 1992, Tercer Mundo Editores, Bogotá, Colombia.

- PROGRAMA de las NACIONES UNIDAS para el DESARROLLO. Informe sobre Desarrollo Humano 1993. Ed. PNUD-CIDEAL, Madrid, 1993.

- PROGRAMA de las NACIONES UNIDAS para el DESARROLLO. Informe sobre Desarrollo Humano 1994. Ed. Fondo de Cultura Económica. México

- PROGRAMA de las NACIONES UNIDAS para el DESARROLLO. Informe sobre Desarrollo Humano 1995. Ed. PNUD-CIDEAL, Madrid, 1993.

- RAMIREZ, Mirta Liliana. (1995). Incidencia de Factores ambientales en la Mortalidad Infantil. Provincia del Chaco,1991. En: Revista Geográfica № 122, Julio-Diciembre 1995.Instituto Panamericano de Geografía e Historia IPGH). México.

- ROFFMAN, Alejandro B. Y ROMERO Luis A. (1973). Sistema Socioeconómico y Estructura Regional en la Argentina. Amorrortu Editores, Buenos Aires.

\section{Fuentes Estadísticas}

- Instituto Nacional de Estadística y Censos - I.N.D.E.C. Censo Nacional de Población y Vivienda 1991. Resultados definitivos, Características Generales, Serie C, Buenos Aires. Provincia de Misiones.

- Instituto Nacional de Estadística y Censos - I.N.D.E.C. Censo Nacional de Población y Vivienda 1991. Resultados definitivos, Características Generales, Serie C, Buenos Aires. Provincia de Formosa.

- Instituto Nacional de Estadística y Censos - I.N.D.E.C. Censo Nacional de Población y Vivienda 1991. Resultados definitivos, Características Generales, Serie C, Buenos Aires. Provincia de Corrientes.

- Instituto Nacional de Estadística y Censos - I.N.D.E.C. Censo Nacional de Población y Vivienda 1991. Resultados definitivos, Características Generales, Serie C, Buenos Aires. Provincia de Chaco 
G Revista Geográfica Digital. IGUNNE. Facultad de Humanidades. UNNE. Año 1- № 2 Julio - Diciembre 2004. ISSN 1668-5180 Resistencia, Chaco

- Instituto Nacional de Estadística y Censos (INDEC) - Dirección de Estadística y Censo (D.E.y C.), Provincia de Corrientes. Situación y Evolución Social Provincial. Corrientes. Síntesis No 1, Buenos Aires, 1997.-

- Instituto Nacional de Estadística y Censos (INDEC) - Instituto Provincial de Estadística y Censo (I.P.E.y C.), Provincia de Misiones. Situación y Evolución Social Provincial. Misiones. Síntesis No 1, Buenos Aires, 1997.-

- Instituto Nacional de Estadística y Censos (INDEC) - Dirección de Estadística y Censo (Provincia del Chaco). Situación y Evolución Social Provincial. Chaco. Síntesis $\mathrm{N}^{\mathrm{o}} 1$, Buenos Aires, 1999.-

- Dirección de Estadística, Censos y Documentación -SE.PLA.DE- Gobierno de la provincia de Formosa. Anuario Estadístico de la provincia de Formosa, 1997.- 
@evista Geográfica Digital. IGUNNE. Facultad de Humanidades. UNNE. Año 1- № 2 Julio - Diciembre 2004. ISSN 1668-5180 Resistencia, Chaco

ANEXO CARTOGRAFICO

SERIE N ${ }^{\circ} 1$
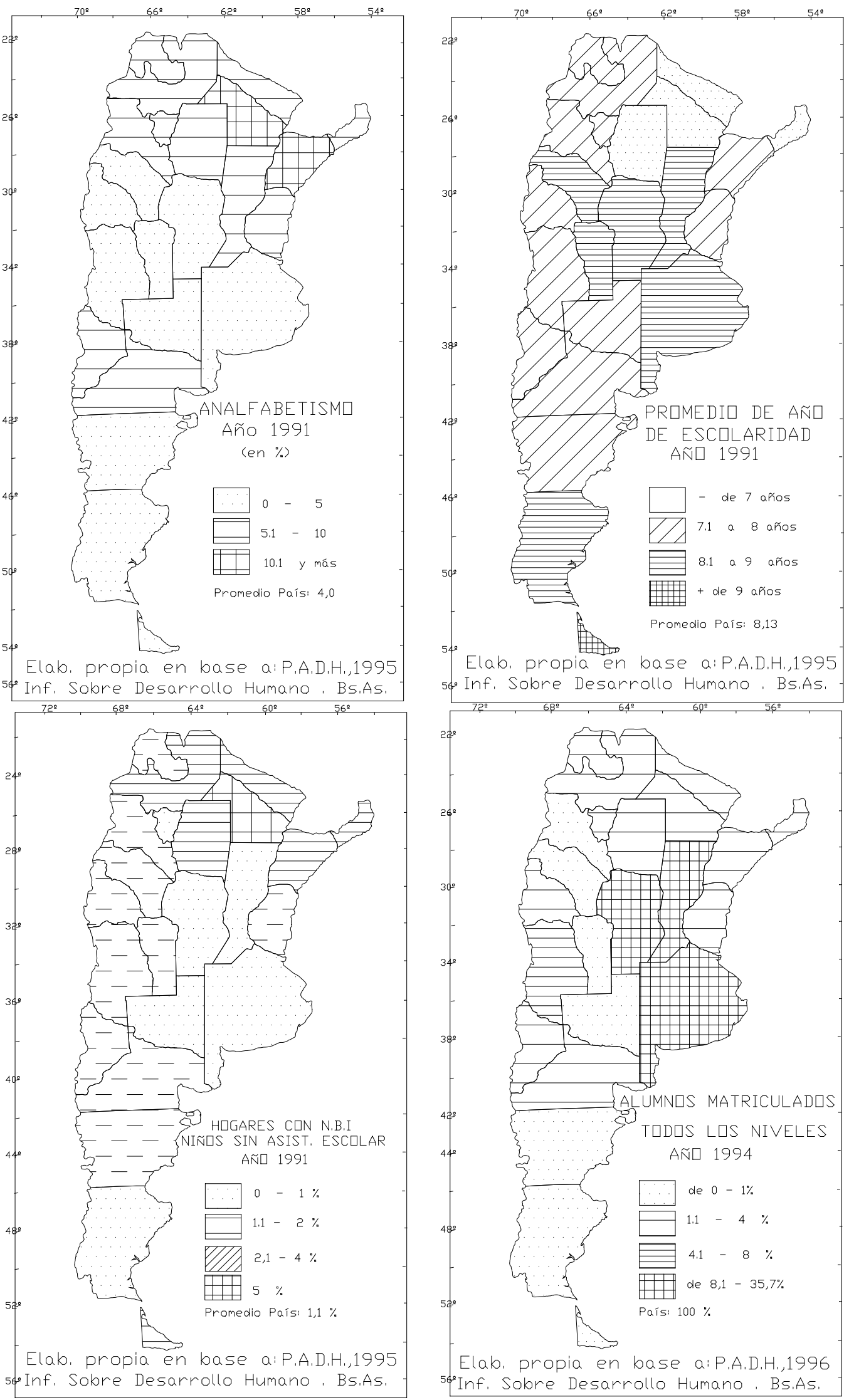

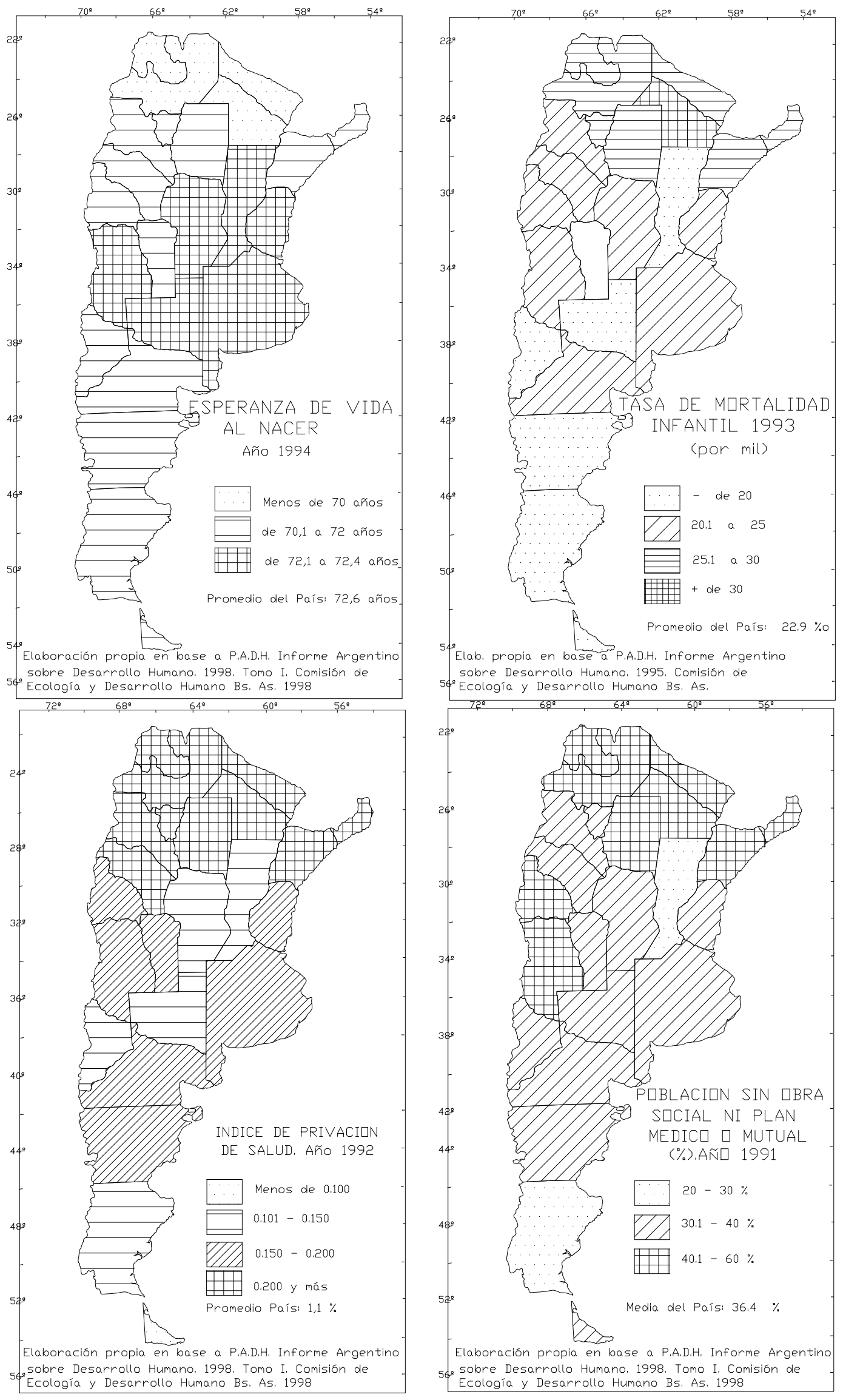

\section{ANEXO CARTOGRAFICO SERIE No 2}

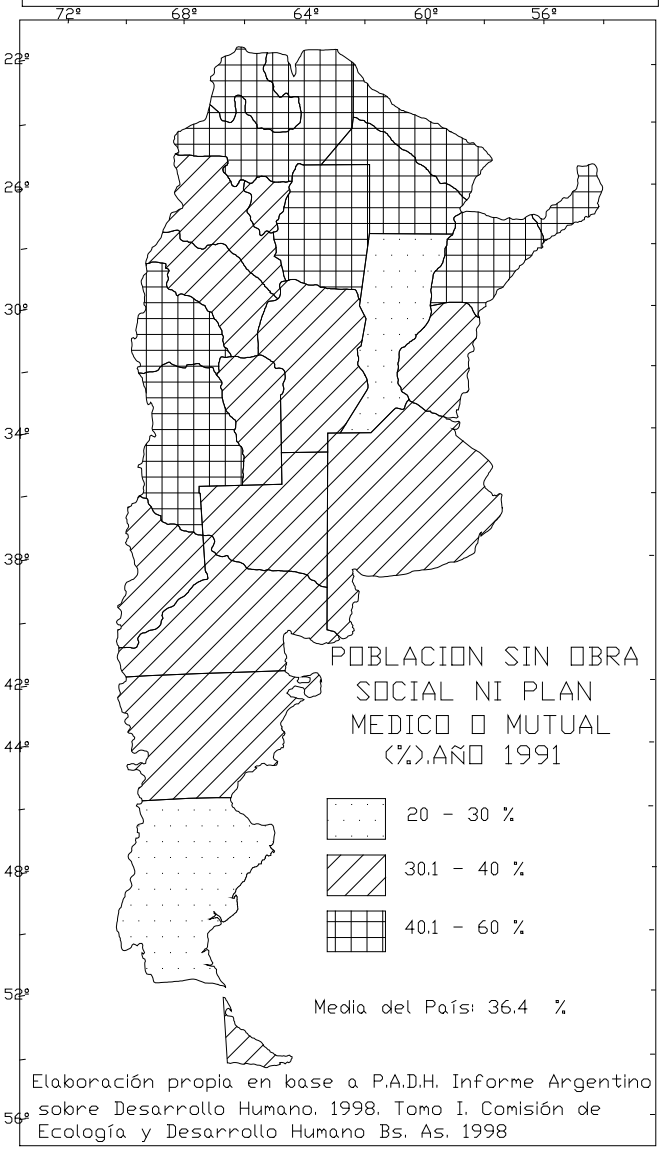


Julio - Diciembre 2004. ISSN 1668-5180 Resistencia, Chaco

\section{ANEXO CARTOGRAFICO} SERIE N 3
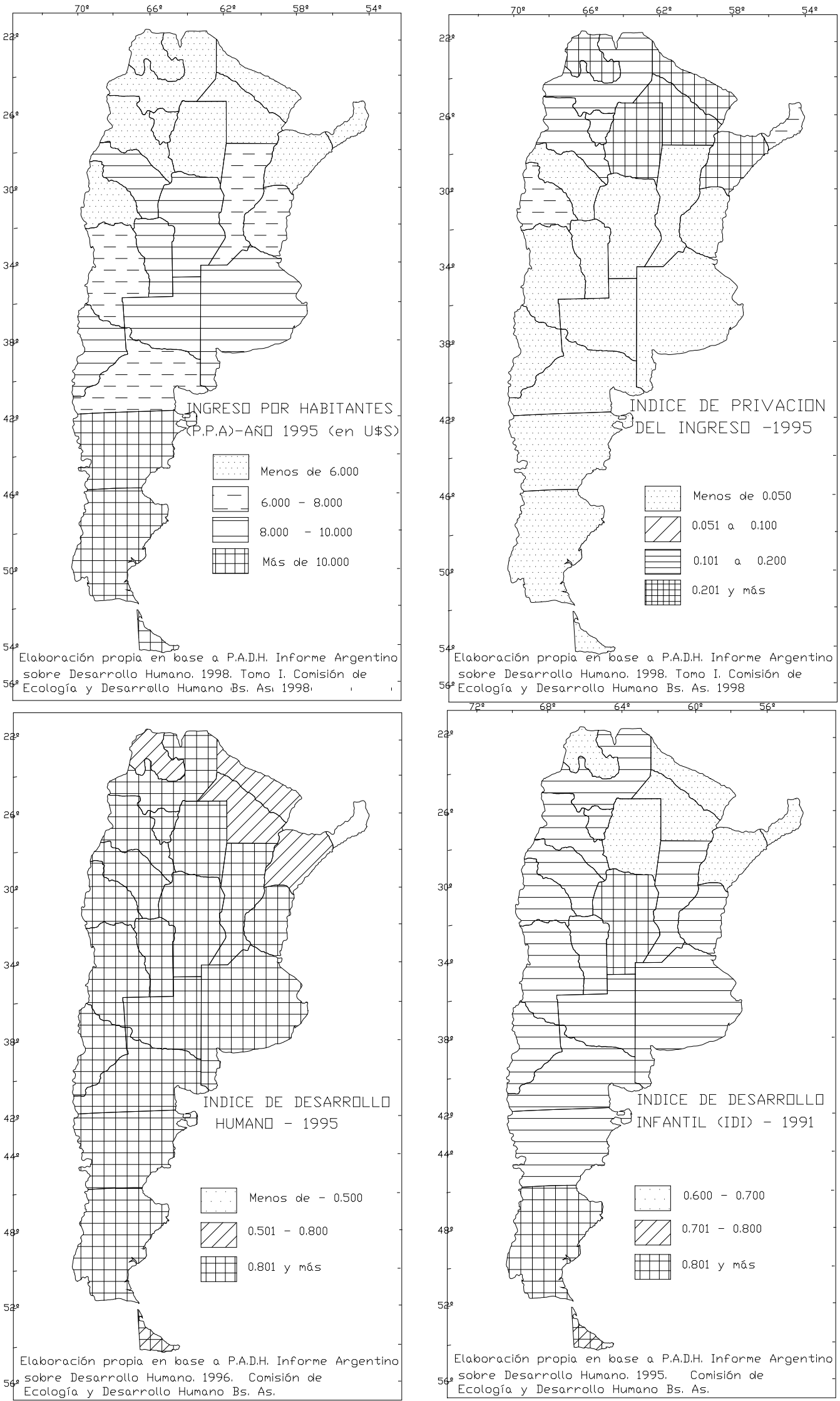